\title{
EGFR mutational status in lung adenocarcinoma: Staging implications or continuous evolution?
}

\author{
Winifred M. Lo, MD, and R. Taylor Ripley, MD
}

\author{
From the Thoracic and GI Oncology Branch, National Cancer Institute, CCR/NIH, Bethesda, Md. \\ Disclosures: Authors have nothing to disclose with regard to commercial support. \\ Received for publication July 16, 2017; accepted for publication July 31, 2017; available ahead of print Sept 1, \\ 2017 \\ Address for reprints: R. Taylor Ripley, MD, Thoracic and GI Oncology Branch, Clinical Research Center, \\ National Cancer Institute, Building 10, 4-3952, 10 Center Dr, MSC 1201, Bethesda, MD 20892-1201 \\ (E-mail: taylor.ripley@nih.gov). \\ J Thorac Cardiovasc Surg 2017;154:1766-7 \\ 0022-5223/\$0.00 \\ Published by Elsevier Inc. on behalf of The American Association for Thoracic Surgery \\ http://dx.doi.org/10.1016/j.jtcvs.2017.07.053
}

Patients with advanced non-small cell lung cancer (NSCLC) with epidermal growth factor receptor (EGFR) mutations (EGFR-mut) have longer progression-free survivals than do those with wild-type EGFR (EGFR-wt). ${ }^{1}$ The prognosis of EGFR-mut in earlier stage disease and whether mutational subtypes have prognostic significance is not as clear. ${ }^{2}$ In their study in this issue of the Journal, Takamochi and colleagues ${ }^{3}$ examined whether EGFR-mut (and if so, which specific EGFR-mut) genotypes have prognostic value after resection for lung adenocarcinoma. They reviewed 939 patients and found that EGFR-mut was associated with longer 5-year overall survival than was EGFR-wt. The individual mutations (exon 21 L858R point mutation [L858R] vs exon 19 deletion [19-del]) were not associated with different survivals.

Takamochi and colleagues ${ }^{3}$ are to be commended for several strengths. First, the number of patients with adequate testing $(\mathrm{n}=939)$ who had adenocarcinoma (45\% EGFR-mut) is noteworthy. Second, exclusion of perioperative therapy helped determine the prognostic role of EGFR-mut without tyrosine kinase inhibitor (TKI) treatment. Takamochi and colleagues ${ }^{3}$ contradict a negative meta-analysis, in which they correctly note that most studies were small and underpowered. Of the 16 studies, however, the largest 2 report that EGFR-mut genotypes were associated with survival advantages. D'Angelo and colleagues report an overall survival advantage in stage I to III adenocarcinoma with EGFR mutations. ${ }^{4} \mathrm{~A}$ significant percentage received adjuvant cytotoxic chemotherapy and EGFR-TKI. In 307 patients, one of these studies reported that EGFR-mut was independently associated with improved overall survival and disease-free survival. No patients received adjuvant TKI or cytotoxic therapy. Despite differences in demographics, staging, and adjuvant treatment, the conclusions of testing and stratification according to EGFR status are relatively similar.

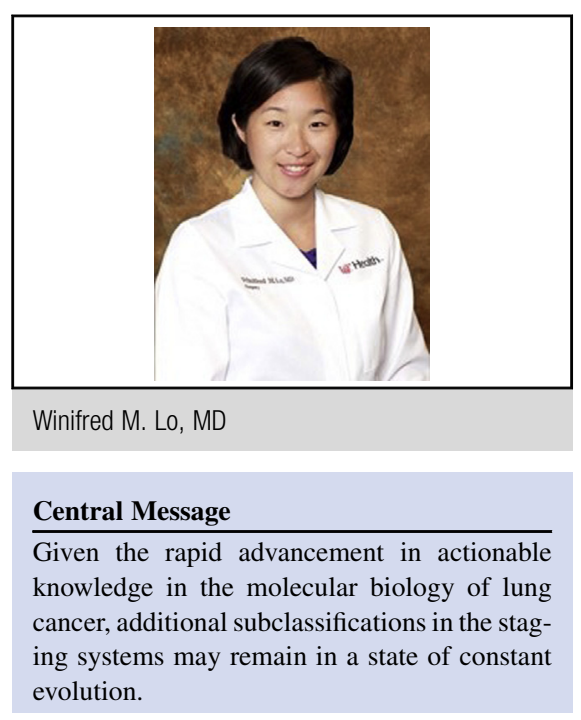

See Article page 1768 .

The ENSURE study (A Study of Erlotinib [Tarceva] Versus Gemcitabine/Cisplatin as First-line Treatment in Patients With Non-Small Cell Lung Cancer With EGFR Mutations) reported a progression-free survival advantage with 19-del compared with L858R for patients treated with erlotinib versus cytotoxic chemotherapy..$^{5}$ A small, retrospective study found improved overall survival with 19-del versus L858R. ${ }^{6}$ Takamochi and colleagues ${ }^{3}$ reported that among 222 patients (53\%) with L858R and 161 patients $(39 \%)$ with 19-del, no survival advantages were observed. Fourteen patients $(3.3 \%)$ of the EGFR-mut genotypes had T790 mutations, which were often associated with additional mutations. The molecular biology underlying EGFR-mut has several clinical implications. First, TKI resistance develops in all patients. The first-generation TKIs reversibly bind to the tyrosine kinase domain, whereas the third-generation TKIs irreversibly bind with more than 100 times the affinity. As studies are reported with third-generation TKIs, the treatment implications and the prognostic value of individual mutations will likely change. Takamochi and colleagues ${ }^{3}$ speculate that "complex oncogenic processes" with multiple mutations are more common in EGFR-wt tumors, unlike EGFR-mut tumors, which rely on a specific driver mutation. Indeed, lung adenocarcinoma has the third highest number of mutations in tumor-normal pair. ${ }^{7}$ In addition, a lower frequency of neoepitopes was observed in patients with 
adenocarcinoma who had never smoked than in those who were smokers. ${ }^{8}$ The implication is that chronic exposure to smoking results in thousands of somatic mutations that likely affect multiple oncogenic pathways, reducing the chance that targeting a single mutation will be effective.

Takamochi and colleagues ${ }^{3}$ recommend that EGFR status be considered in future staging guidelines. Given the rapid advancement in TKI therapy and the increases in actionable knowledge in the molecular biology of lung cancer, additional subclassifications in the staging systems may remain in a state of constant evolution that will rarely, if ever, be adequately reflected in staging guidelines.

\section{References}

1. Fukuoka M, Wu YL, Thongprasert S, Sunpaweravong P, Leong SS, Sriuranpong V, et al. Biomarker analyses and final overall survival results from a phase III, randomized, open-label, first-line study of gefitinib versus carboplatin/paclitaxel in clinically selected patients with advanced non-small-cell lung cancer in Asia (IPASS). J Clin Oncol. 2011;29:2866-74.
2. Zhang Z, Wang T, Zhang J, Cai X, Pan C, Long Y, et al. Prognostic value of epidermal growth factor receptor mutations in resected non-small cell lung cancer: a systematic review with meta-analysis. PLoS One. 2014;9: e106053.

3. Takamochi K, Oh S, Matsunaga T, Suzuki K. Prognostic impacts of EGFR mutation status and subtype in patients with surgically resected lung adenocarcinoma. $J$ Thorac Cardiovasc Surg. 2017;154:1768-74.e1.

4. D'Angelo SP, Janjigian YY, Ahye N, Riely GJ, Chaft JE, Sima CS, et al. Distinct clinical course of EGFR-mutant resected lung cancers: results of testing of 1118 surgical specimens and effects of adjuvant gefitinib and erlotinib. J Thorac Oncol. 2012;7:1815-22.

5. Wu YL, Zhou C, Liam CK, Wu G, Liu X, Zhong Z, et al. First-line erlotinib versus gemcitabine/cisplatin in patients with advanced EGFR mutation-positive non-small-cell lung cancer: analyses from the phase III, randomized, open-label, ENSURE study. Ann Oncol. 2015;26: 1883-9.

6. Riely GJ, Pao W, Pham D, Li AR, Rizvi N, Venkatraman ES, et al. Clinical course of patients with non-small cell lung cancer and epidermal growth factor receptor exon 19 and exon 21 mutations treated with gefitinib or erlotinib. Clin Cancer Res. 2006;12:839-44.

7. Lawrence MS, Stojanov P, Polak P, Kryukov GV, Cibulskis K, Sivachenko A, et al Mutational heterogeneity in cancer and the search for new cancer-associated genes. Nature. 2013;499:214-8.

8. Campbell JD, Alexandrov A, Kim J, Wala J, Berger AH, Pedamallu CS, et al Distinct patterns of somatic genome alterations in lung adenocarcinomas and squamous cell carcinomas. Nat Genet. 2016;48:607-16. 